\title{
Evolution in Fine-Grained Environments. II. Habitat Selection as a Homeostatic Mechanism
}

\author{
Alan R. Templeton \\ Department of Biology, Washington Untversity, St. Louis, Missouri 63130
}

\author{
AND \\ EDWARD D. ROTHMAN
}

Department of Statistics, University of Michigan, Ann Arbor, Michigan 48109

Received September 21, 1979

\begin{abstract}
A model of genotype specific habitat selection is developed for an organism subject to within-lifetime environmental fluctuations. Habitat selection is first overlaid upon both hard and soft selection Levene models with either discrete or continuous habitats. It is shown that even if all genotypes have identical physiological and fitness responses within a habitat, habitat selection can still maintain a polymorphism. In other words, physiological divergence is not a necessary prerequisite for divergence in habitat preferences. Within-lifetime environmental variability is then assumed to occur within each chosen habitat. It is shown that habitat selection acts as an evolutionary filter that can enhance the fitness impact of some niches and effectively eliminate the impact of others such that it generally increases the chances for a polymorphism under soft selection. However, density-dependent effects obscure the relationship between physiological fitness and evolutionary outcome. Indeed, it is possible for selection to favor an allele causing its bearers to preferentially go to the niche to which they are least physiologically adapted. Hence. changes in habitat preference can evolve before an organism has completely adapted physiologically to a new habitat. The fitness impact of habitat selection interacts with both homeostatic avoidance mechanisms (i.e., short-term buffering) and with tolerance (long-term) mechanisms. In general, habitat selection will be most favored in those organisms deficient in long-term tolerance. Moreover, habitat selection tends to accentuate selection favoring short-term avoidance mechanisms. Thus, organisms displaying much habitat selection should have poor physiological long-term tolerances but effective physiological short-term avoidance mechanisms. Finally, if the fitness costs associated with habitat selection are too large to be ignored and are comparable for all genotypes, habitat selection directs the selective pressures back onto the physiological homeostatic capabilities. Hence, the very existence and extent of habitat selection depends critically upon the physiological capabilities of the organism.
\end{abstract}


Most organisms must survive and reproduce in environments that can vary both in space and time. To cope with such environmental heterogeneity. organisms divert some of their energy and resources into various homeostatic mechanisms that buffer them against environmental uncertainty. In the first paper of this series (Templeton and Rothman, 1978a), we considered how organisms respond to temporal heterogeneity within their lifetime by evolving physiological avoidance and/or tolerance to extreme exposures of various environmental states. In this paper, we overlay spatial heterogeneity upon this temporal variability. Spatial heterogeneity allows yet another type of homeostasis (fitness buffering) to evolve-habitat selection.

The are several cases in the literature in which different genotypes or phenotypes within a species distribute themselves non-randomly throughout a spatially heterogeneous environment or display some form of habitat selection (Taylor and Powell, 1977 and 1979; Tabachnick and Powell, 1978; Kettlewell, 1955; Richmond and Gerking, 1979; Nevo et al.. 1979; Cox and Cox, 1974; see also Mayr, 1963, p. 246ff).

For example, Christensen (1977) has shown that viability among amylase genotypes in the isopod Asellus aquaticus is influenced by the number of days of frosty weather during the proceeding winter. However, the pond environment in which these isopods live is not uniform, and Christensen performed experiments that demonstrated that specific amylase genotypes preferred and selectively migrated to different habitats within the pond. The critical observation in this data is that habitat migration is genotype specific. Hence, high viability in frosty weather could be achieved by selecting for the appropriate habitat preference. In this sense, habitat selection could function as a type of homeostatic mechanism in these animals.

Another example is provided by the work of Cavener (1979) on preference for ethanol level by larvae of Drosophila melanogaster as a function of their alcohol dehydrogenase (ADH) locus genotype. ADH has been implicated in tolerance to ethanol stress in Drosophila, and Cavener discovered that larvae with the highest genotypic tolerance (ADH "fast" homozygotes) were preferentially found in food with high ethanol levels when offered a choice, whereas the less tolerant "slow" homozygotes were not. Once again, this illustrates an interaction between habitat selection and physiological homeostasis (tolerance to ethanol stress) in which both attributes are apparently influenced by the same locus (Cavener, 1979).

Habitat selection, however, is not limited to animals. Many plants have variability in seed traits that greatly affect the dispersal of the seeds, which can be viewed as a potential type of habitat selection. Perhaps more importantly, many plants have delayed germination of their seeds (Templeton and Levin, 1979). Quite often, the germination of the seeds is preferentially cued by certain environmental features. This creates a situation that is selectively analogous to an animal choosing among spatial patches. Indeed, the 
evolutionary genetic analysis of delayed germination in annuals given in Templeton and Levin (1979) depends upon a matrix whose form is similar to a model of spatial heterogeneity given by Karlin (1976). Consequently, the models we develop in this paper are relevant to both plants and animals.

\section{A Levene Model with Genotype Specific Habitat Selection}

In the original Levene model and subsequent elaborations (e.g., see Karlin, 1976), it is usually assumed that the dispersal pattern is genotype independent. Thus, any "habitat selection" that does arise is due to an interaction of the habitat specific selective forces operating across generations with the genotype independent migration parameters (e.g., Maynard Smith, 1966) and is not due to active choices by individuals of a specified genotype searching out a specific type of habitat. We now develop a Levene-type model with an infinite population mating at random and then dispersing over several habitats. However, we allow individuals to actively chose a habitat by letting $\gamma_{j}(i)$ be the proportion of zygotes of genotype $j$ going to habitat $i$, where $\sum_{i} \gamma_{j}(i)=1$ for all $j$.

Suppose we have a single locus with two alleles, $A$ and $a$, and let $W_{j}(i)$ be the fitness of genotype $j$ in niche $i$. Then, given an initial allele frequency $p$ of $A$, the frequency of $A$ in habitat $i$ after selection, $p_{i}^{\prime}$, is given by

$$
p_{i}^{\prime}=\left[\gamma_{A A}(i) p^{2} W_{A A}(i)+\gamma_{A a}(i) p q W_{A a}(i)\right] / \bar{W}(i),
$$

where

$$
\bar{W}(i)=\gamma_{A A}(i) p^{2} W_{A A}(i)+2 \gamma_{A a}(i) p q W_{A a}(i)+\gamma_{a a}(i) q^{2} W_{a a}(i) .
$$

I et $k_{i}$ be the proportion of adults contributed to the total population from habitat $i$. We initially examine the soft selection case in which $k_{i}$ is a constant; that is, the proportion of the adult population coming from the $i$ th habitat is independent of the genotypic composition entering that habitat. Such a constant $k_{i}$ represents the relative carrying capacity of habitat $i$ with respect to the total carrying capacity summed over all habitats. The fact that the $k$ 's are independent of genotypic composition implies the $k$ 's are determined by density-dependent ecological constraints and not genetic ones, and moreover that the density-dependent constraints operate independently for each habitat. Under these conditions, for $A$ rare

$$
p^{\prime} \simeq p \sum_{i} k_{i} \cdot \frac{\gamma_{A a}(i) W_{A a}(i)}{\gamma_{a a}(i) W_{a a}(i)}
$$


Therefore, $A$ will be protected from loss whenever

$$
\bigvee_{i} k_{i} \cdot \frac{\gamma_{A a}(i) W_{A a}(i)}{\gamma_{a a}(i) W_{a a}(i)}>1
$$

Similarly, for $a$ to be protected from loss

$$
\frac{\bigvee}{i} k_{i} \cdot \frac{\gamma_{A a}(i) W_{A a}(i)}{\gamma_{A A}(i) W_{A A}(i)}>1
$$

An interesting special case arises when $W_{J}(i)=W(i)$ for all $j$; that is, no relative fitness differences exist in any habitat. (This would occur if the physiological responses of all genotypes to the habitat environment were the same.) In this case, (1.3) and (1.4) reduce to

$$
\searrow^{\prime} k_{i} \gamma_{A a}(i) / \gamma_{j}(i)>1, \quad j=A A \text { and } a a \text {. }
$$

Thus, even with identical fitness responses to all niches, a polymorphism can be maintained with habitat selection. As can be seen from (1.5), this occurs when the heterozygotes are the most efficient at choosing those habitats with high carrying capacities $\left(k_{i}\right.$ 's).

A hard selection analogue of this model can be constructed by setting

$$
k_{i}=\bar{W}(i) / \frac{1}{i} \bar{W}(i),
$$

that is, the contribution of habitat $i$ to the total population depends upon the number of zygotes entering habitat $i$ and their average fitnesses in habitat $i$ at the locus of interest. In this case, the $k$ 's vary as the genotypic composition entering the habitat varies, and the relative output from a given habitat is determined solely by this entering genotypic composition and the absolute fitnesses of the genotypes. Coupling (1.6) with (1.1), we have

$$
p^{\prime}=\searrow_{i} k_{i} p_{i}^{\prime}=\left[p^{2} \searrow_{\gamma_{A A}}(i) W_{A A}(i)+p q \searrow_{\gamma_{A a}}(i) W_{A a}(i)\right] / \bigcup_{\bar{W}}(i)
$$

Hence, the conditions for a polymorphism are

$$
\sum_{A a}(i) W_{A a}(i)>\sum_{j}(i) W_{j}(i), \quad j=A A \text { and } a a
$$

Note, that when $W_{j}(i)=W(i)$ for all genotypes, (1.8) can still be satisfied if heterozyotes are most efficient at choosing those habitats in which their absolute fitness is large. Hence, the ability of habitat selection to maintain a 
polymorphism when all genotypes have identical physiological responses does not depend critically upon the assumption of soft selection.

We now consider briefly a continuous analogue to the above that is modeled after Taylor $(1975,1976)$. Suppose the organisms mate at random and then disperse over a continuous environment. We assume the environment can be described by a continuous probability distribution $\phi(X)$, where $X$ describes the state of the environment at a particular point ( $X$ may be a vector). Let $W_{j}(X, N)$ be the absolute fitness of genotype $j$ at point $X$ with density $N$. Then, Taylor (1976) has shown that sufficient conditions for the maintenance of a genetic polymorphism under random dispersal are

$$
\int \phi(X)\left[W_{A a}\left(X, N_{j}\right)-W_{j}\left(X, N_{j}\right)\right] d X>0, \quad j=A A \text { and } a a
$$

Taylor $(1975,1976)$ also considered the conditions for polymorphism under "optimal habitat selection" in which individuals occupy those habitats where they are most fit. The conditions for polymorphism then become that there exist points $X_{1}$ and $X_{2}$ with $\phi>0$ such that

$$
\begin{aligned}
& K_{A a}\left(X_{1}\right)>K_{A A}\left(X_{1}\right), \\
& K_{A a}\left(X_{2}\right)>K_{a a}\left(X_{2}\right),
\end{aligned}
$$

where $K_{j}(X)$ is defined implicitly by $W_{j}\left(X, K_{j}(X)\right)=1$.

In Taylor's formulations $\phi$ describes an abundance distribution over environmental states. However, $\phi$ is really the effective species-specific environmental distribution that takes into account how the species of interest perceives and exploits its environment. When there is genetic variability within a species, we can let $\phi_{j}(X)$ describe the weighting assigned to environment $X$ by genotype $j$ due to the action of habitat selection (Templeton and Rothman, 1978b). Then, sufficient conditions for the maintenance of a polymorphism are

$$
\begin{gathered}
\int \phi_{A a}(X) W_{A a}\left(X, N_{j}\right) d X>\int \phi_{j}(X) W_{j}\left(X, N_{j}\right) d X, \\
j=A A \text { and } a a .
\end{gathered}
$$

Once again, note that $(1.11)$ can be satisfied when $W_{j}(X, N)=W(X, N)$ for all genotypes. However, (1.10) cannot be satisfied under those conditions, so if all genotypes are capable of making "optimal" habitat choices a polymorphism can only be maintained if there are fitness differences in at least some habitats. In this sense, non-optimal habitat selection broadens the conditions for polymorphism over Taylor's optimal habitat selection model. 


\section{Habitat Selection and Homeostasis in a Discrete Habitat Levene Model}

In the previous section we simply assumed the organisms were capable of shifting the random dispersal distribution into a non-random distribution. However, in general such a shift will cost the organism some fitness. The organism might have to divert energy from survival and reproduction into morphological, sensory and neurological traits that allow effective habitat selection. Moreover, habitat selection often implies some period of sampling the environment, and during this period the individuals are expending energy and may be more exposed to predation or extreme environments. Consequently, we now add a fitness cost onto the habitat selection. Moreover, we must now define how the habitat selection relates to environmental parameters and physiological homeostasis.

The environmental model within a chosen habitat is identical to that described in Templeton and Rothman (1978a). At any given time, the environment is in one of two possible states, 0 or 1 . Transitions between time units are governed by the stochastic matrix

$$
\begin{aligned}
& 0 \quad 1 \\
& \left.0 \quad \begin{array}{cc}
1-\alpha & \alpha \\
\beta & 1-\beta
\end{array}\right),
\end{aligned}
$$

where $1-\alpha$ is the probability of being in state 0 given state 0 during the previous time unit, $\alpha$ is the probability of being in state 1 given 0 previously, $\beta$ is the probability of being in state 0 given 1 previously, and $1-\beta$ is the probability of being in state 1 given 1 previously. Matrix (2.1) is assumed to describe the temporal transitions occurring in a specific habitat or point. Hence, in the discrete habitat models, each habitat is characterized by the parameter pair $\left(\alpha_{i}, \beta_{i}\right)$. In the continuous model, the spatial heterogeneity in the environment is described by a continuous probability distribution over $\alpha$ and $\beta, \phi(\alpha, \beta)$.

Once an organism has chosen a habitat or location in the environment, we assume it lives for $L$ more time units at the chosen place, reproduces and dies. During this period, the viability of the organism depends upon its responses to the encountered states of 0 and 1 , and in particular upon the run lengths of 0 's or 1's (i.e., the durations of continuous exposures to 0 's or 1's). Since the emphasis in this paper is on habitat selection, we consider only a special case of the more generalized fitness models given in Templeton and Rothman (1978a), although the results can be readily extended to these other fitness models. In this paper, we assume genotype $j$ is sensitive only to runs of 1 's such that the fitness response given it has already settled in a habitat characterized by specific values for $\alpha$ and $\beta$, $R_{j}(\alpha, \beta)$, is given by 


$$
R_{j}(\alpha, \beta)=\prod_{s} \omega_{j r_{s}},
$$

where $s$ indexes all runs of 1's experienced during $L$ and $\omega_{j r}$ is the fitness response of genotype $j$ to a run of 1's of length $r$ such that

$$
\begin{array}{ll}
\omega_{j r}=1 & \text { if } \quad r \leqslant d_{j}, \\
\omega_{j r}=e^{-\lambda_{j}\left(r-d_{j}\right)} & \text { if } \quad r>d_{j} .
\end{array}
$$

The parameters $d_{j}$ represents the short-term physiological avoidance capabilities, whereas $\lambda_{j}$ represents the long term tolerance to more prolonged exposures to state 1 (Templeton and Rothman, 1978a). We further assume that $L$ is much larger than $1 / \alpha+1 / \beta$ so that the variance in fitness at the individual level may be ignored (Templeton and Rothman, 1978a). Under these conditions, $R_{j}$ converges to a constant given by (Templeton and Rothman, 1978a)

$$
R_{j} \rightarrow E\left(R_{j}\right) \simeq \exp \left[-L \alpha \lambda_{j}(1-\beta)^{d_{j}} /(\alpha+\beta)\right] .
$$

$R_{j}(\alpha, \beta)$ would be the fitness of genotype $j$ in a habitat characterized by $\alpha$ and $\beta$ if there were no cost to the act of habitat selection. However, suppose there is a fitness cost to individuals of genotype $j$ in finding and settling in their ultimate habitats of choice. Then, letting $C_{j}$ reflect the fitness efficiency of habitat selection, the total fitness is

$$
W_{j}=C_{j} R_{j}(\alpha, \beta)
$$

Note that as $C$ decreases, the fitness cost of habitat selection increases. We also take the convention that $C_{j}=1$ if genotype $j$ randomly disperses over the possible habitats. Hence, $C_{j}$ is a function of both the amount of effort a particular genotype invests in the act of habitat selection as well as the fitness costs (discussed earlier) that may accompany such an effort.

We must now overlay spatial heterogeneity upon this fitness model for temporal heterogeneity at a given location. For the discrete habitat Levene model with habitat selection, coupling (2.6) with (1.4) and (1.5) yields that a polymorphism will be protected when

$$
\sum_{i} k_{i} \frac{\gamma_{A a}(i) R_{A a}\left(\alpha_{i}, \beta_{i}\right)}{\gamma_{j}(i) R_{j}\left(\alpha_{i}, \beta_{i}\right)}>\frac{C_{j}}{C_{A a}}, \quad j=A A \text { and } a a
$$

Inequality (2.7) reveals several interesting features of the model. First, the evolutionary outcome is greatly influenced by the relative efficiencies of habitat selection for the various genotypes. If, for example, the heterrozygote is more efficient than the homozygotes, $C_{j} / C_{A a}<1$, the conditions for a 
protected polymorphism are broadened. On the other hand, if $C_{j}>C_{A a}$. it is more difficult to maintain a protected polymorphism.

Second, habitat selection will often result in a positive correlation between the $\gamma$ 's and the $R$ 's. In particular, suppose there are habitats in which some genotypes do well (i.e., high $R$ 's) and others poorly, so that the ratio of $R_{A a} / R_{j}$ is either high or low, depending upon the habitat. Habitat selection will often accentuate the tendency of this ratio to be either high or low; that is, the ratio $\gamma_{A a} R_{A a} /\left(\gamma_{j} R_{j}\right)$ will tend to be even larger or smaller than the original ratio $R_{A a} / R_{j}$. Hence, habitat selection acts as an evolutionary filter that enhances the fitness impact of some habitats and effectively eliminates the impact of other habitats-a phenomenon analogous to the temporal filtering of the environment caused by delayed germination in plants (Templeton and Levin. 1979). Moreover, the nature of this filter is such that it would generally increase the chances for a polymorphism.

The evolutionary weighting of the environments is also influenced by $k_{i}$, the relative carrying capacity of the $i$ th habitat. If the heterozygote has large relative fitness (i.e., $R_{A a} / R_{j}>1$ ) in those habitats with large carrying capacities, the chances for a polymorphism are enhanced and habitat selection will further enhance it. However, an interesting situation arises when the heterozygote has its highest fitness responses in environments with low $k$ 's. Suppose, for example, that three environments exist with $k_{1}=0.1$. $k_{2}=0.2$ and $k_{3}=0.7, \quad$ and $R_{A a}(1) / R_{j}(1)=2, \quad R_{A a}(2) / R_{j}(2)=1 \quad$ and $R_{A a}(3) / R_{j}(3)=0.5$ for $j=A A$ and $a a$. Without habitat selection, the lefthand side of Eq. (2.7) equals 0.75 , which is less than 1 and hence the polymorphism is not protected (with no habitat selection. we assume $C_{j}-1$ for all genotypes). Now suppose the heterozygotes only go to environment 3 , but the homozygotes equally disperse over all three environments. Then $\gamma_{A a}(3) / \gamma_{j}(3)=3$ and Eq. (2.7) equals 1.05 , which will protect a polymorphism as long as $C_{A a}>0.95$. In other words, if $A$ is rare and causes heterozygotes to go to that environment to which they are least adapted physiologically relative to the homozygotes, $A$ can persist in the population. whereas if the $A$ allele did not affect habitat choice it would be lost! Thus. the presence of density-dependent, soft-selection effects obscures the relationships between physiological fitness and evolutionary outcome. Hence, "optimal" physiological adaptations may be difficult to define in an evolutionary sense in some circumstances. The above example also shows how natural selection can favor an organism switching to or specializing on a habitat before it has completely adapted physiologically to that habitat when density-dependent factors override in fitness importance the physiological constraints. This phenomenon is analogous to that occurring between temporal fluctuations in relative fitness and absolute seed crop production in plants with delayed germination, as discussed in Templeton and Levin (1979). 
We now briefly turn our attention to the hard selection, discrete habitat model. Substituting (2.6) into (1.8) yields the conditions for a polymorphism to be

$$
\frac{\sum \gamma_{A a}(i) R_{A a}\left(\alpha_{i}, \beta_{i}\right)}{\sum \gamma_{j}(i) R_{j}\left(\alpha_{i}, \beta_{i}\right)}>\frac{C_{j}}{C_{A a}} .
$$

The same arguments about the costs of habitat selection apply in this model as in the soft-selection case. However, the fitness filtering aspect of habitat selection has, in general, less impact on (2.8) than on (2.7). In (2.7), the filter operated directly on the ratios $R_{A a} / R_{j}$ and tended to broaden conditions for a polymorphism because an $x \%$ increase in a large ratio more than compensates for an $x \%$ decrease in a small ratio. However, $(2.8)$ depends on the ratio of the averages rather than the average of the ratios. Habitat selection will in general cause both the numerator and the denominator to increase. Thus, although habitat selection can maintain a polymorphism in the absence of heterosis or even any physiological fitness differences among genotypes in any single habitat, habitat selection should in general be less effective in maintaining polymorphisms under hard selection than under soft selection.

\section{Interactions between Habitat Selection and Physiological Homeostasis}

We now examine more closely the interactions of habitat selection with physiological homeostasis. These interactions are most clearly illustrated by looking at the impact of habitat selection on log-fitness, which under the assumption of $L$ much larger than $1 / \alpha+1 / \beta$ is a monotonically increasing function of physiological fitness. From (2.6) and (2.4), the log fitness is

$$
\ln C_{j}-L \alpha \lambda_{j}(1-\beta)^{d_{j}} /(\alpha+\beta) \text {. }
$$

To examine the relationship between habitat selection and the physiological parameters, $\lambda$ and $d$, that enter into (3.1), we need a more explicit definition of habitat selection. There are several specific models of habitat selection one could envision. For example, one simple model is to assume that an organism samples habitats until it finds one in state 0 (since state 1 is associated with deleterious fitness responses). The degree of habitat selection exercised by a specific genotype could then be described by how many failures (i.e., the number of habitats in state 1) it will sample before settling in a habitat regardless of state. If we assume all habitats are sampled independently with respect to a stationary stochastic process described by 
(2.1), the probability of a habitat characterized by $\alpha$ and $\beta$ being in state 0 is $\beta /(\alpha+\beta)$. Hence, if a genotype will accept $n$ failures before settling regardless of habitat state, the original distribution over $\alpha$ and $\beta, f(\alpha, \beta)$ (either discrete or continuous) is modified into

$$
\frac{p_{0}\left(1+p_{1}+p_{1}^{2}+\cdots+p_{1}^{n-1}\right) \beta f(\alpha, \beta) /(\alpha+\beta)+p_{1}^{n}(\alpha, \beta)}{p_{0}^{2}\left(1+p_{1}+p_{1}^{2}+\cdots+p_{1}^{n-1}\right)+p_{1}^{n}},
$$

where $p_{0}=1-p_{1}=\int_{0}^{1} \int_{0}^{1} \beta f(\alpha, \beta) /(\alpha+\beta) d \alpha d \beta$. As is evident from (3.2), the greater the amount of habitat selection (as measured by $n$ ), the more the habitats with large $\beta$ 's are favored. In general, we will assume that what ever the details of the habitat selection mechanics are, its overall result is to favor habitats with $\beta$ large (that is, the probability of the habitat being in state 1 is small, or alternatively, the probability of a state 1 following another $1,1-\beta$, is small). This assumption follows from the fact that since the organisms are sensitive to runs of l's, it is reasonable to assume they will choose environments in which they do well physiologically. Although this seems reaonable, the example provided in Section 2 should be kept in mind. There may be circumstances in which this is not an appropriate criterion for habitat choice.

Given that habitat selection tends to make $\beta$ large, we can gauge the sensitivity of $\log$ fitness to habitat choice induced changes in $\beta$ by taking the partial derivative of 3.1 with respect to $\beta$ to yield

$$
\frac{\partial \ln C_{j}}{\partial \beta}+\frac{L \alpha \lambda_{j}(1-\beta)^{d_{j}}}{\alpha+\beta}\left[\frac{d_{j}}{1-\beta}+\frac{1}{\alpha+\beta}\right] .
$$

In general, we assume $\partial \ln C_{j} / \partial \beta$ is negative; that is, as habitat selection causes $\beta$ to increase more and more, the costs of making the habitat choice also increase. However, for now we will concentrate on the second term in (3.3). As can be seen from this term, the fitness impact of habitat selection depends upon both physiological parameters, $\lambda$ and $d$, but most strongly on $d$. If $d$ is very large (i.e., the organism has very effective physiological avoidance mechanisms) $(1-\beta)^{d_{j}}$ will be small for any $\beta$ less than 1 , and the augmenting of log fitness by habitat selection will be minimal. However, as $d_{j}$ becomes smaller, $(1-\beta)^{d_{j}}$ increases, and the sensitivity of $\log$ fitness to changes in $\beta$ also increases, particularly since the term $d_{j} /(1-\beta)$ increases as habitat selection increases. Habitat selection therefore interacts most strongly with the physiological avoidance parameter. Indeed, habitat selection itself can be regarded as a homeostatic avoidance mechanism. However, habitat selection should not be regarded as an alternative to $d$. As can be seen from Eq. (3.3) log fitness is most sensitive to moderate values of $d$ when $1-\beta$ is small. Hence, habitat selection will if anything increase the 
intensity of selection favoring the increase of $d$ to moderate levels. Thus, habitat selection coupled with short-term physiological avoidance mechanisms is an extremely effective homeostatic strategy for increasing individual fitness. Moreover, the combination of effective habitat selection coupled with moderate $d$ 's greatly reduces the necessity for effective long term physiological tolerance mechanisms (i.e., small $\lambda$ 's). In fact, from (3.3) it is obvious that those organisms with the least effective tolerance mechanisms ( $\lambda$ large) will benefit most from habitat selection. Therefore, if habitat selection is an option, a large $\lambda$ would create strong selection for habitat selection, and effective habitat selection would diminish the intensity of any selection favoring reductions in $\lambda$. In summary we would predict that those organisms displying much habitat selection would have poor long-term physiological tolerances but effective short-term physiological avoidance mechanisms.

To further investigate these relationships, we consider the continuous habitat model for which the conditions for protection are given by 1.11 . We assume there are no density-dependent effects (i.e., hard selection). With our assumption that $L$ is much larger than $1 / \alpha+1 / \beta, 1.13$ will be true if

$$
\int \dot{\phi}_{A a}(X) \ln W_{A a}(X) d X>\int \phi_{j}(X) \ln W_{j}(X) d X
$$

Hence, the evolutionary outcome is determined by the average over the environment of the log fitnesses. We now consider a special case in which the average frequency of state $1, \alpha /(\alpha+\beta)$, is fixed at $p_{1}$ and all effects of habitat selection occur strictly through $\beta$ subject to the constraint of $p_{1}$ fixed; that is, the average state frequencies are constant throughout the environment, but there is heterogeneity in the chances for runs of 1's over the habitat space. In this case, $\phi$ can be regarded strictly as a probability distribution over $\beta$. Since $\beta$ can vary from 0 to 1 , a convenient choice of $\phi$ is the beta distribution

$$
\phi_{j}(\beta)=\frac{\Gamma\left(N_{j}\right)}{\Gamma\left(u_{j}\right) \Gamma\left(v_{j}\right)} \beta^{u_{j}-1}(1-\beta)^{v_{j}-1},
$$

where $N_{j}=u_{j}+v_{j}$ and $\Gamma()$ is the gamma function. Suppose under random dispersal

$$
\phi_{r}(\beta)=\frac{\Gamma(M)}{\Gamma(a) \Gamma(b)} \beta^{a-1}(1-\beta)^{b-1},
$$

where $M=a+b$. The mean value of $\beta$ is $a / M$ under random dispersal and $u_{j} / N_{j}$ under habitat selection and the variances are $a b /\left[M^{2}(M+1)\right]$ and 
$u_{j} v_{j} /\left[N_{j}^{2}\left(N_{j}+1\right)\right]$, respectively. The expected log fitnesses of the form given by 3.1 are

$$
\begin{aligned}
E_{r}\left(\ln W_{j}\right) & =-L p_{1} \lambda_{j} \frac{\Gamma(M) \Gamma\left(b+d_{j}\right)}{\Gamma(b) \Gamma\left(M+d_{j}\right)} \\
& =-L p_{1} \lambda_{j} \frac{b(b+1) \cdots\left(b+d_{j}-1\right)}{M(M+1) \cdots\left(M+d_{j}-1\right)}
\end{aligned}
$$

for random dispersal, and

$$
E_{j}\left(\ln W_{j}\right)=\ln C_{j}-L p_{1} \lambda_{j} \frac{v_{j}\left(v_{j}+1\right) \cdots\left(v_{j}+d_{j}-1\right)}{N_{j}\left(N_{j}+1\right) \cdots\left(N_{j}+d_{j}-1\right)}
$$

with habitat selection. It will be to the advantage of genotype $j$ (in terms of (3.4)) to engage in habitat selection whenever (3.8) is greater than (3.7). In the special case of no $\operatorname{cost}\left(\ln C_{j}=0\right)$ and with $M=N_{j}$, this inequality reduces to

$$
v_{j}<b,
$$

that is, the average value of $1-\beta$ with habitat selection must be less than that for random dispersal.

Given the constraint that $N_{j}=M$, habitat selection can be measured in terms of decreasing values of $v_{j}$ (or increasing values of $-v_{j}$ ). Hence, we can examine the interaction of habitat selection with the physiological parameters with the following partial derivative

$$
\frac{\partial E\left(\ln W_{j}\right)}{\partial\left(-v_{j}\right)}=\frac{\partial \ln C_{j}}{\partial\left(-v_{j}\right)}+L p_{1} \lambda_{j} \frac{v_{j}\left(v_{j}+1\right) \cdots\left(v_{j}+d_{j}-1\right)}{M(M+1) \cdots\left(M+d_{j}-1\right)} \cdot \sum_{i=0}^{d_{j}-1} \frac{1}{v_{j}+i} .
$$

Once again, we assume $\partial \ln C_{j} / \partial\left(-v_{j}\right)$ is negative. From (3.10), we can draw the same qualitative conclusions as from (3.3); namely, that habitat selection interacts most directly with the short-term physiological avoidance parameter $d_{j}$; that when $v_{j}$ is small (effective habitat selection), fitness is greatly augmented by a moderate value of $d_{j}$, but as $d_{j}$ increases, the fitness return rapidly drops off; that $v_{j}$ small with $d_{j}$ moderate results in a very effective fitness homeostasis even in organisms that have poor physiological tolerance $(\lambda$ large).

By setting (3.10) equal to zero, we can identify that value of $v_{j}$ that maximizes average $\log$ fitness. As long as $\partial \ln C_{j} / \partial\left(-v_{j}\right)<0$, this "optimal" $v_{j}$ will always occur at some $v_{j}>0$; that is, not all the individuals of genotype $j$ will be at points that maximize their physiological fitness. This definition of "optimal" is not necessarily contradictory with Taylor's (1975, 
1976) since his model had no cost. Using (3.10) to define "optimal habitat selection" leads to an interesting conclusion when $\partial \ln C_{j} / \partial\left(-v_{j}\right)$ is the same for all genotypes. For example, suppose in all genotypes the fitness decline resulting from engaging in habitat selection depends upon the amount of decrease in $1-\beta$ induced by habitat selection from $1-\beta_{r}$, the expected value of $1-\beta$ under random dispersal, such that

$$
\ln C_{j}=-c\left[1-\beta_{r}-\left(1-\beta_{j}\right)\right]=-c\left(b-v_{j}\right) / M .
$$

Then, $\partial \ln C_{j} / \partial\left(-v_{j}\right)=-c / M$. Thus, if all gnotypes engage in optimal habitat selection in the sense defined above, each genotype will have a $v_{j}$ such that

$$
E\left(\ln W_{j}\right)=-\frac{c}{M}\left[b-v_{j}-\left(\sum_{i=0}^{d_{j}-1} \frac{1}{v_{j}+i}\right)^{-1}\right] .
$$

Note that all fitness differences are due to $d_{j}$ and $v_{j}$ and do not depend explicitly on $\lambda_{j}$. This reinforces our earlier conclusion that habitat selection interacts most directly with the short-term avoidance mechanisms (measured by $d_{j}$ ) in determining fitness, and that habitat selection diminishes the fitness importance of long-term tolerance (measured by $\lambda_{j}$ ). This is not to say, however, that long-term tolerance is now totally unimportant for $v_{j}$ is implicitly a function of both $d_{j}$ and $\lambda_{j}$. For example, if $v_{j}$ and $M$ are very large (the variance in $\beta$ in the environment is low), then the optimal $v_{j} / M$ defined by $(3.10)$ is approximately

$$
\frac{v_{j}}{M} \simeq\left(\frac{c}{L p_{1} \lambda_{j} d_{j}}\right)^{1 /\left(d_{j}-1\right)} .
$$

Thus, as $\lambda_{j}$ increases, fitness decreases under optimal habitat selection. Effectively, $\lambda_{j}$ is an important determinant of how much effort the organism should invest in habitat selection even though its direct effects upon fitness are masked by optimal habitat selection. Moreover, since $v_{j}$ is implicitly a function of $\lambda_{j}$ and $d_{j}$, all selection is ultimately redirected upon the physiological homeostatic capabilities of the organism as long as the fitness cost of habitat selection cannot be ignored. Thus, habitat selection with cost does not eliminate selection on physiological homeostasis, but rather alters the fitness weighting assigned to short-term versus long-term homeostatic mechanisms.

\section{Discussion}

The models and examples presented in this paper have shown that the interactions between habitat selection and physiological homeostasis are 
varied and complex, and that a number of paradoxes or difficulties seemingly arise. First, a low cost habitat selection can effectively become the primary mode of homeostasis in an organism and free the organism from selection on its physiological homeostatic capabilities. But if non-negligible costs exist that are similar for all genotypes in the sense defined in Section 3 and if all genotypes display "optimal habitat selection" (i.e., they maximize their average fitness), habitat selection directs all the selective pressures onto the physiological homeostasis capabilities. Hence, one should not always regard habitat selection as an alternative to physiological homeostasis for often the very existence of effective habitat selection depends critically upon the physiological capabilities of the organism. Also, not all physiological homeostatic mechanisms interact in the same manner with habitat selection. Short-term avoidance mechanisms interact most directly with habitat selection with respect to fitness such that effective habitat selection coupled with moderate physiological avoidance capabilities obviates the need for long-term physiological tolerance to prolonged exposures to deleterious environmental states.

Second, our models reveal that under some circumstances habitat selection acts as an evolutionary filter that accentuates the differences in physiological fitness among the genotypes, yet, it would be a mistake to conclude that genotypic differences in physiological homeostasis are a necessary prerequisite for the evolution of habitat selection. As we have shown in this paper, genotypic specific habitat selection can evolve and be maintained by both hard and soft selection in the total absence of differences in physiological responses among the genotypes. Hence, the demonstration that genotypes display different habitat preferences cannot in general be used to infer that the genotypes differ in their physiological fitness responses.

Finally, we demonstrated that density-dependent factors can obscure the relationship between habitat selection and physiological responses. For example, in Section 2 we examined a case in which natural selection favors an allele causing its bearers to preferentially go to the habitat to which they were least physiologically adapted. This observation immediately leads to difficulties in defining what is "optimal habitat selection." It also points out the difficulty of making adaptive interpretations of physiological data in the absence of knowledge about the existence of habitat selection and its costs and the role of density dependent factors in the population in its natural setting. This observation also illustrates that selection can favor an organism to shift its niche or specialize before it has become physiologically adapted to the habitat under certain density-dependent constraints. Presumably. once such a habitat preference shift has been made. subsequent selection would operate upon the physiological capabilities of the organisms, but the physiological adaptation would be a secondary effect of a habitat shift and not a primary cause. 
A proper understanding of the role of habitat selection as a homeostatic mechanism will require studies not only detailing the nature of the habitat selection and the environmental variability, but also studies investigating the fitness costs of habitat selection, the physiological constraints of the organism, and the role of density dependent and density independent factors in regulating population size in the various habitats.

\section{ACKNOWLEDGMENTS}

We wish to thank Dr. Charles Taylor and the anononyous reviewers for their critical comments on an earlier draft of this manuscript. This work was supported in part by N.S.F. Grant DEB 78-10455.

\section{REFERENCES}

Cavener, D. 1979. Preference for ethanol in Drosophila melanogaster associated with the alcohol dehydrogenase polymorphism, Behavior Genetics 9, 359-365.

Christensen, B. 1977. Habitat preference among amylase genotypes in Asellus aquaticus (Isopoda, Crustacea), Hereditas 87, 21-26.

Cox, G. W. AND Cox, D. G. 1974. Substrate color matching in the grasshopper Circolettix rabula (Orthoptera: Acrididae), Great Basin Nat. 34, 60-70.

KARLIN, S. 1976. Population subdivision and selection migration interaction, in "Population Genetics and Ecology" (S. Karlin and E. Nevo, Eds.), Academic Press, New York.

KETTLEWELL, H.B.D. 1955. Recognition of appropriate backgrounds by the pale and black phase of Lepitoptera, Nature 175, 934.

MAYR, E. 1963, "Animal Species and Evolution," Belknap, Cambridge.

Nevo, E., R, Guttman, Haber, M., AND Erez, E. 1979. Habitat selection in evolving mole rats, E cologia 43, 125-138.

Tabachnick, W. J. and Powell, J. R. 1978. Genetic structure of the East African domestic populations of Aedes aegypti, Nature 272, 535-537.

TAYLOR, C. E. 1975. Genetic loads in heterogeneous environments, Genetics 80, 621-635.

TAYIOR, C. F. 1976. Genetic variation in heterogenenus environments, Genetics 83, 887-894.

TAYLOR, C. E. AND POWELL, J. R. 1977. Microgeographic differentiation of chromosomal and enzyme polymorphisms in Drosophila persimilis, Genetics 85, 681-695.

TAYLOR, C. E. AND Powell, J. R. 1978. Habitat choice in natural populations of Drosophila, Ecologia 37, 69-75.

Templeton, A. R. ANd Levin, D. A. 1979. Evolutionary consequences of seed pools, Amer. Nat. 114, 232-249.

Templeton, A. R. AND Rothman, E. D. 1978a. Evolution in fine-grained environments. I. Environmental runs and the evolution of homeostasis, Theor. Pop. Biol. 13, 340-355.

Templéton, A. R. ANd Rothman, E. D. 1978b. Evolution and fine-grained environmental runs, in "Foundations and Applications of Decision Theory, Volume II: Epistemic and Social Applications" (C. A. Hooker, J. J. Leach and E. F. McClennen, Eds.), Reidel, Dordrecht, Holland. 